\title{
Aportes Metodológicos para el Diseño de Sistemas de Supervisión de Procesos Continuos
}

\author{
Germán Zapata $^{(1)}$, Juan Cardillo ${ }^{(2)}$ y Edgar Chacón ${ }^{(2)}$ \\ (1) Universidad Nacional de Colombia, Facultad de Minas, Cra. 80 № 65-223, Medellín-Colombia. \\ (e-mail: gdzapata@unal.edu.co) \\ (2) Universidad de Los Andes, Facultad de Ingeniería - Núcleo Universitario La Hechicera, \\ Mérida-Venezuela (e-mail: ijuan@ing.ula.ve, echacon@ula.ve)
}

Recibido Jun. 30, 2010; Aceptado Sep. 06, 2010; Versión Final recibida Oct. 26, 2010

\begin{abstract}
Resumen
Se revisan los conceptos sobre la supervisión de procesos y se define la terminología y las funciones básicas de un sistema supervisor, así como los diferentes enfoques para el diseño de dicho sistema. Se selecciona un formalismo para la representación y modelado de la dinámica del proceso y se expresan las especificaciones desde esa base formal. La propuesta metodológica está orientada a los sistemas convencionales, pero la representación formal de la especificación y el modelo del comportamiento sirven de base para agregar las funcionalidades propias de sistemas modernos de supervisión inteligente.
\end{abstract}

Palabras clave: supervisión de procesos, supervisión industrial, control supervisorio, automatización integrada

\section{Methodological Contributions for the Design of Supervision Systems of Continuous Processes}

\begin{abstract}
The concepts of supervised process available in the literature are reviewed and the terminology and the basic functions of a supervisory system are defined. Also, the different approaches used for the design of such systems are analyzed. An appropriate formalism for the representation and modeling of the dynamics of the process is selected and the system specifications are expressed from that formal selected basis. The proposed methodology is focused on the conventional hierarchical systems, but both, the formal representation of the specification as well as the behavior model can be used for adding the other functionalities of other modern intelligent supervision systems.
\end{abstract}




\section{INTRODUCCION}

La función denominada "Supervisión del proceso", dentro del entorno de la automatización e integración de procesos industriales, adquiere mayor importancia a medida que los enfoques muestran tendencias hacia sistemas inteligentes, integrados y distribuidos, como los denominados Manufactura inteligente (IMS) y los Sistemas de manufactura holónicos (HMS). Como un paso previo que conduzca al diseño de sistemas de supervisión holónicos sobre bases formales se requiere disponer de metodologías que permitan expresar las especificaciones del sistema, modelar el comportamiento del proceso controlado y supervisado, y probar las propiedades exigidas de desempeño. En este trabajo se proponen algunos elementos metodológicos para la especificación, modelamiento y diseño de sistemas de supervisión convencionales y que sirven de base para abordar la concepción de la supervisión de los procesos industriales desde el enfoque holónico. Es claro que para construir esta base, además de precisar los objetivos y funciones de la supervisión, se deben unificar y precisar términos y definiciones que pueden crear confusión.

En la práctica industrial e incluso en la académica, se usan indistintamente los términos "supervisión de procesos", "supervisor", "control supervisorio", "control supervisado". Igualmente los términos "monitoreo", "SCADA" tienen diferentes interpretaciones. Existen diferencias en estos conceptos dependiendo de la dinámica del sistema: si es continua, discreta o híbrida. El presente trabajo se centra en procesos de producción con dinámicas continuas, entre los que pueden referenciarse sistemas de generación de vapor, generación de energía eléctrica, acueductos, gasoductos, estaciones de bombeo, refinerías, destilación y otros procesos en la industria química y de alimentos. La necesidad de "supervisar" un proceso industrial aparece cuando difícilmente un operador puede deducir las condiciones que permitan mantener el funcionamiento normal, incluso en condiciones de falla, debido a la cantidad de variables, la complejidad del proceso, la distribución y heterogeneidad de las variables, comportamientos y subprocesos (Contreras et al., 2001). Generalmente la supervisión del proceso se refiere a actividades de toma de decisiones, dirigidas a mantener el funcionamiento del sistema. El operador identifica las diversas condiciones operativas y adelanta acciones apropiadas (Waissman, et al., 2000) apoyado por el sistema de supervisión (Montmain y Gentil, 1999).

En la literatura se encuentran múltiples definiciones para la función de supervisión. Por ejemplo, en la función es la coordinación y selección de los puntos de operación de los lazos de control con el fin de mantener las variables en puntos óptimos (Wang, et al., 2003; Eigi Miyagi, et al., 2001; Blanke, et al., 2001; Quek y Wahab, 2000). Jantzen (1998) y Yazdi (1997) hacen énfasis en el desempeño de los controladores locales, definiendo un sistema supervisorio como aquel que avalúa si los controladores locales satisfacen criterios de desempeño especificados, diagnostican causas de desviación del desempeño, planean acciones correctivas y las ejecutan. Stobart y Shadbolt (1990) exigen a los sistemas de monitoreo y control, mantener alta eficiencia, advirtiendo ante fallas de componentes o caídas de eficiencia. Se afirma también que la contribución de un sistema de supervisión es mejorar la seguridad y confiabilidad del sistema a ser supervisado (Villa, et al., 2003; Vagelatos, et al., 2001; Isermann, 1997). Se encuentran también enfoques desde la calidad (Fiol-Roig y Ferrer-Gili, 1997) o desde el planeamiento reactivo (Ya-Li, et al., 2004; Gonnet y Chiotti, 1999). Mención especial merece el enfoque de Saridis (Saridis, 1988; Saridis y Valavanis, 1986), quien formula el problema de supervisión como "la secuencia de decisiones en un sistema jerárquico estructurado según el principio de incrementar precisión y disminuir inteligencia, que minimiza la entropía total del sistema". Uraikul, et al.(2007) y Waissman, et al. (2000) mencionan como tareas del sistema de supervisión, el monitoreo de datos del proceso, el análisis de estado actuales, la detección y diagnóstico de anomalías del proceso y ejecución de acciones de control apropiadas. Así mismo, para Jantzen (1998), Vagelatos, et al.(2001) e Isermann (1997), las tareas típicas del control supervisorio son operación segura y estable, alta calidad de productos y operación económica.

La vigilancia de rangos de variables es otra función que aparece en (Villa, et al., 2003). El control de secuencias, los procedimientos de arranque y parada se presentan como funciones del supervisor en (Kowalewski, et al., 1999) donde se plantean además las funciones de evitar estados no deseados o daño de equipos. Muchos trabajos y propuestas enfocan el problema de 
supervisión desde el diagnóstico de fallas (FD): (Montmain y Gentil, 1999; Esteva Payet, 2003). La tendencia del énfasis en FD dio origen al denominado Control Tolerante a Fallos (FTC) (Blanke, et al., 2001; Kwang-Hyun y Jong-Tae, 1998; Izadi-Zamanabadi y Blanke, 1999). Desde este enfoque "el objetivo final de la supervisión es el de lograr sistematizar la tolerancia a las fallas. Otra parte importante es la ayuda en la toma de decisiones con el propósito de disminuir los efectos de las fallas en el funcionamiento del proceso". La tolerancia a fallos se entiende como la capacidad de un sistema de control para mantener los objetivos de control a pesar de la aparición de un fallo, admitiéndose una cierta degradación de sus prestaciones (Puig, et al., 2004).

\section{DISEÑO DE SISTEMAS DE SUPERVISIÓN}

Para abordar el diseño de sistemas de supervisión, es importante diferenciar claramente y clasificar la dinámica predominante del sistema: si es de eventos discretos (DES), de variable continua (CVS) o híbrido (HS). Coinciden muchos autores en afirmar que las dinámicas en el nivel de supervisión son de naturaleza discreta (Cassandras y Lafortune, 2008; Alvarez, et al., 1999; Raisch y O'Young, 1998; Garcia, 1997), así el proceso sea de variable continua. En estos procesos, la dinámica continua puede ser alterada por acciones discretas. Por esto, muchos de estos autores abordan el problema desde la Teoría de control supervisorio (SCT) propuesta por Ramadge y Wonham (Ramadge y Wonham, 1984). Desde DES, (Rezg y Niel, 1996) y (KwangHyun y Jong-Tae, 1998) plantean que el concepto de supervisor está asociado al concepto de controlador, haciendo una analogía con la teoría de control realimentado en CVS. En este sentido, la función de supervisión está asociada a generar acciones de control para habilitar y deshabilitar eventos controlables, con el objetivo de tener un desempeño del sistema en lazo cerrado, tal que la trayectoria de eventos esté siempre en un conjunto de cadenas de eventos deseados. En (Lunze, et al., 2001) se obtiene una representación discreta de la evolución de los estados del proceso continuo, mediante cuantificadores. En (Rezg y Niel, 1996) se aborda la supervisión desde SCT, mediante Redes de Petri orientadas a objetos. Desde DES se genera una política de control que garantiza que se evita el estado prohibido y se permite un máximo conjunto de transiciones de estado.

Desde estas concepciones, al pretender abordar el estudio de los sistemas de supervisión para procesos continuos, no deben desconocerse los aportes de los sistemas de eventos discretos y de la Teoría de control supervisorio, dada la naturaleza de las dinámicas presentes en el nivel de supervisión y el enfoque desde la teoría de control. Los sistemas actuales muestran tendencias hacia los denominados Sistemas de supervisión integrados (Quek y Wahab, 2000) y los sistemas de supervisión inteligentes: (Jantzen, 1998; Lo, et al., 2006; Quek y Ng, 1996; Isermann, 1998; Rezg, et al., 1997). Estos sistemas incluyen nuevas funcionalidades, tales como, seguridad de la planta, manejo de la disponibilidad, diagnóstico en línea y políticas de mantenimiento (Montmain y Gentil, 1999). Los sistemas basados en arquitecturas de agentes cooperantes, que se fundamentan en los avances de la computación distribuida y la solución distribuida de problemas, están siendo introducidos en aplicaciones de supervisión de procesos industriales, como sistemas de apoyo a los operadores en tareas como diagnóstico de fallas y restablecimiento del sistema (Avouris, et al., 1993; Contreras, et al., 2001).

La aparición del paradigma holónico y de los sistemas multiagente también ha influenciado el diseño de los sistemas de supervisión en dos sentidos. Por un lado, integrando los conceptos holónicos o MAS en el diseño de los supervisores (Avouris, et al., 1993; Villa, et al., 2003), en procesos controlados con técnicas convencionales y por otro, abordando el diseño de los supervisores en sistemas en los que se adopta el paradigma holónico para su control (Simão, 2005). La tendencia entonces, desde el enfoque holónico, es tener sistemas distribuidos, basados en la noción de una misión a cumplir, incluyendo algunos grados de libertad, autonomía local, habilidades de cooperación y capacidades de anticiparse a situaciones que pongan en riesgo la misión. La noción de objetivo y su indicador de desempeño asociado se vuelven importantes (Rezg, et al., 1997). 


\section{TERMINOLOGÍA}

Con base en la bibliografía revisada, se extraen las siguientes definiciones que permiten clarificar algunos conceptos relacionados con el tema, tales como: 1.- sistema de supervisión: sistema; 2.control supervisorio; 3.- control supervisado; 4.- scada, supervisory control and data acquisition; y 5.- monitoreo: recolección de datos desde el proceso..

1.- Sistema de supervisión: Sistema con la habilidad de medir un proceso y actuar en él, formado por componentes interactivos que razonan acerca del comportamiento del proceso para proponer y ejecutar acciones apropiadas para mantener las condiciones de operación normal aún caso de fallas. (Contreras, et al., 2001)

2.- Control supervisorio: es una teoría general para la síntesis de controladores, llamados supervisores, en sistemas de eventos discretos (Morgenstern et al., 2007; Akesson et al., 1999). También se aplica a los sistemas continuos cuando el supervisor se diseña desde los sistemas de eventos discretos (DES). El supervisor de un DES cambia la entrada de control de acuerdo al estado actual del sistema, observa su estado y por cada estado admisible, una estrada de control debe ser aplicada en ese punto de funcionamiento. Un evento es admisible para un DES supervisado, si y solo si es físicamente posible y autorizado por la función de supervisión. (Rezg, et al., 1997)

3.- Control supervisado: proceso sometido a un subsistema de control que interactúa directamente con las señales físicas restringiendo su comportamiento y una entidad de orden jerárquico superior que coordina las tareas del controlador. El control supervisado presupone una estructura jerárquica en la que en el bajo nivel se tiene un sistema controlado (discreto o continuo) y en el alto nivel un supervisor que actúa sobre el sistema controlado, presentando como ventaja que el supervisor sólo maneja la coordinación y no considera control directo de los subsistemas (Ver figura 1) (Charbonnier, et al., 1999; Basile y Chiacchio, 2007; Oltean, et al., 2000).

4.- SCADA, Supervisory control and data acquisition: según ISA (International Society of Automation) es la tecnología que habilita a un usuario para recoger datos desde una o más instalaciones distantes y le permite enviar instrucciones de control limitadas a esas instalaciones (Boyer, 2004). Se privilegia en estos sistemas la captura de datos, los sistemas de comunicaciones (sistemas dispersos geográficamente) y mínimas opciones de control. Los SCADA requieren que un operario permanezca al frente del sistema o que visite frecuentemente la ubicación remota (Boyer, 2004). La definición de la IEEE (IEEE 100, 2000) establece que los SCADA son sistemas que operan con señales codificadas sobre canales de comunicación a fin de proporcionar control remoto de equipos, obteniendo información sobre el estado de éstos para la visualización o registro de sus funciones. Puede concluirse entonces que los SCADA son sistemas de adquisición de datos y que junto con un operador humano u otro sistema de toma de decisiones, conforman un sistema de supervisión en el sentido de la definición dada anteriormente. (Ver figura 2)

5.- Monitoreo: Recolección de datos desde el proceso. Determina el estado actual del sistema controlado y hace inferencias necesarias para producir datos adicionales como históricos 0 diagnóstico. El monitoreo se limita a la captura de datos del proceso y no tiene acciones directas en los modelos o en la evolución del estado (Da Silveira y Combacau, 2006; Lambert, et al., 1999; Isermann, 1997, 1998; Suárez, et al., 2003). 


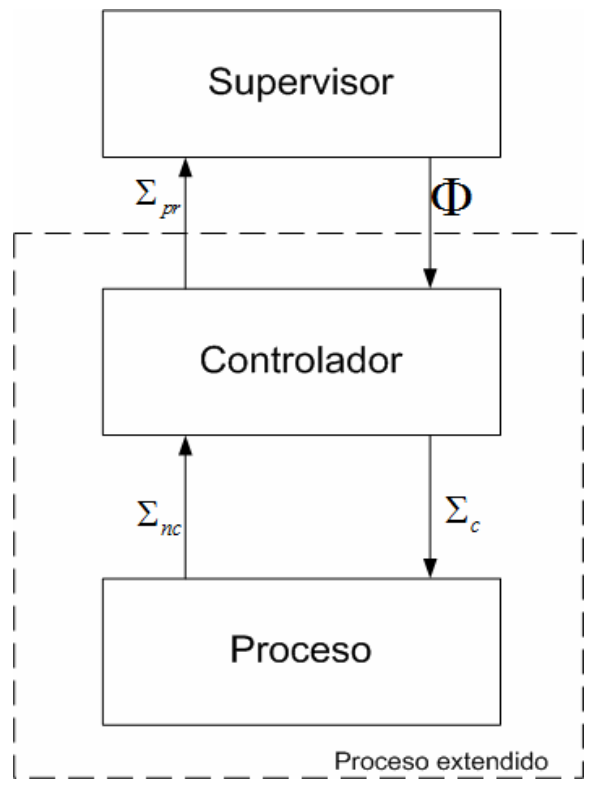

Fig. 1: Control Supervisado

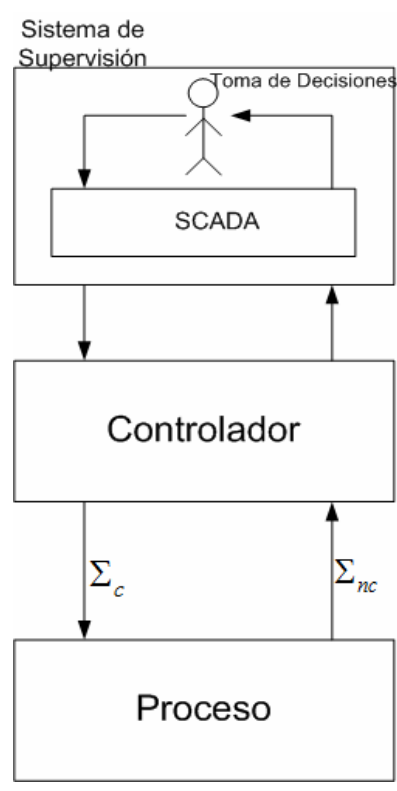

Fig. 2: Sistema de Supervisión

Las funciones de supervisión se pueden clasificar en las siguientes categorías:

Funciones asociadas a las acciones sobre controladores locales determinadas por usuarios o sistemas de niveles superiores, entre las que se encuentran: los procedimientos de arranque y parada y la selección de modos de operación.

Funciones asociadas a la selección y ajuste de leyes de control: determinación de regiones en las cuales opera el sistema, selección y conmutación de leyes de control según región de operación y criterios de desempeño esperados.

Funciones asociadas a la respuesta ante fallas y comportamiento anormal: detección, diagnóstico, aislamiento de fallas, determinación de las condiciones de operación anormal, activación y ejecución de procedimientos de respuesta a situaciones anormales, reconfiguraciones del sistema en caso de falla.

\section{PROPUESTA METODOLÓGICA}

La propuesta metodológica que se presenta, se enmarca dentro de los sistemas integrados de automatización y control. Desde una concepción jerárquica, estos sistemas se componen de un nivel de gestión, un nivel de coordinación, un nivel de supervisión y un nivel de control. En la figura 3 se muestra la estructura jerárquica y se indican las características de los niveles. En los sistemas holónicos estos niveles de decisión se descentralizan, conformando « holones » que se gestionan, coordinan, supervisan y controlan. Con base en lo que se ha planteado se presenta una propuesta metodológica para el diseño de sistemas de supervisión de procesos de producción continua con las siguientes consideraciones:

Las dinámicas presentes en el nivel de decisión del supervisor son discretas, así el proceso sea de variables continuas (Cassandras y Lafortune, 2008; Raisch y O'Young, 1998; Garcia, 1997). Esta afirmación posibilita el uso de técnicas de síntesis de supervisores propias de los sistemas discretos, como la teoría de autómatas y las redes de Petri. El diseño mediante estos métodos formales facilita además la validación de propiedades como la alcanzabilidad, la estabilidad y la controlabilidad. Las dinámicas continuas y las dinámicas discretas se pueden acoplar mediante cuantificadores o detectores de eventos, los cuales permiten obtener imágenes discretas del proceso continuo. Tanto la teoría de autómatas como las redes de Petri comprenden extensiones útiles para la representación de este comportamiento: el autómata híbrido y las redes de Petri híbridas o diferenciales. La arquitectura del supervisor incluye bloques constitutivos acordes con 
las funciones de supervisión establecidas: Autómata, diagnosticador, cuantificador o detector de eventos y conmutador de leyes de control. La plataforma tecnológica existente debe garantizar el flujo de información entre los diferentes niveles de control, supervisión y coordinación.

Los aportes metodológicos se resumen en los siguientes aspectos: (a) Presentación de una base teórica unificada para representar las dinámicas del nivel de proceso y las de los niveles de supervisión y coordinación. (b) La representación formal de las especificaciones del sistema de supervisión, a partir de las cuales se crean los modelos de los sistemas de control y supervisión. La elaboración de estas especificaciones está orientada hacia tener modelos distribuidos de los sistemas, lo cual es consistente con el enfoque holónico. (c) La presentación de una arquitectura que involucra aspectos relacionados con las nuevas tendencias en supervisión de procesos y que son aportes preliminares para la construcción de sistemas de supervisión holónicos. (d) Se relacionan los aspectos formales (modelos) con los aspectos tecnológicos (Interfaces de operador, SCADA).

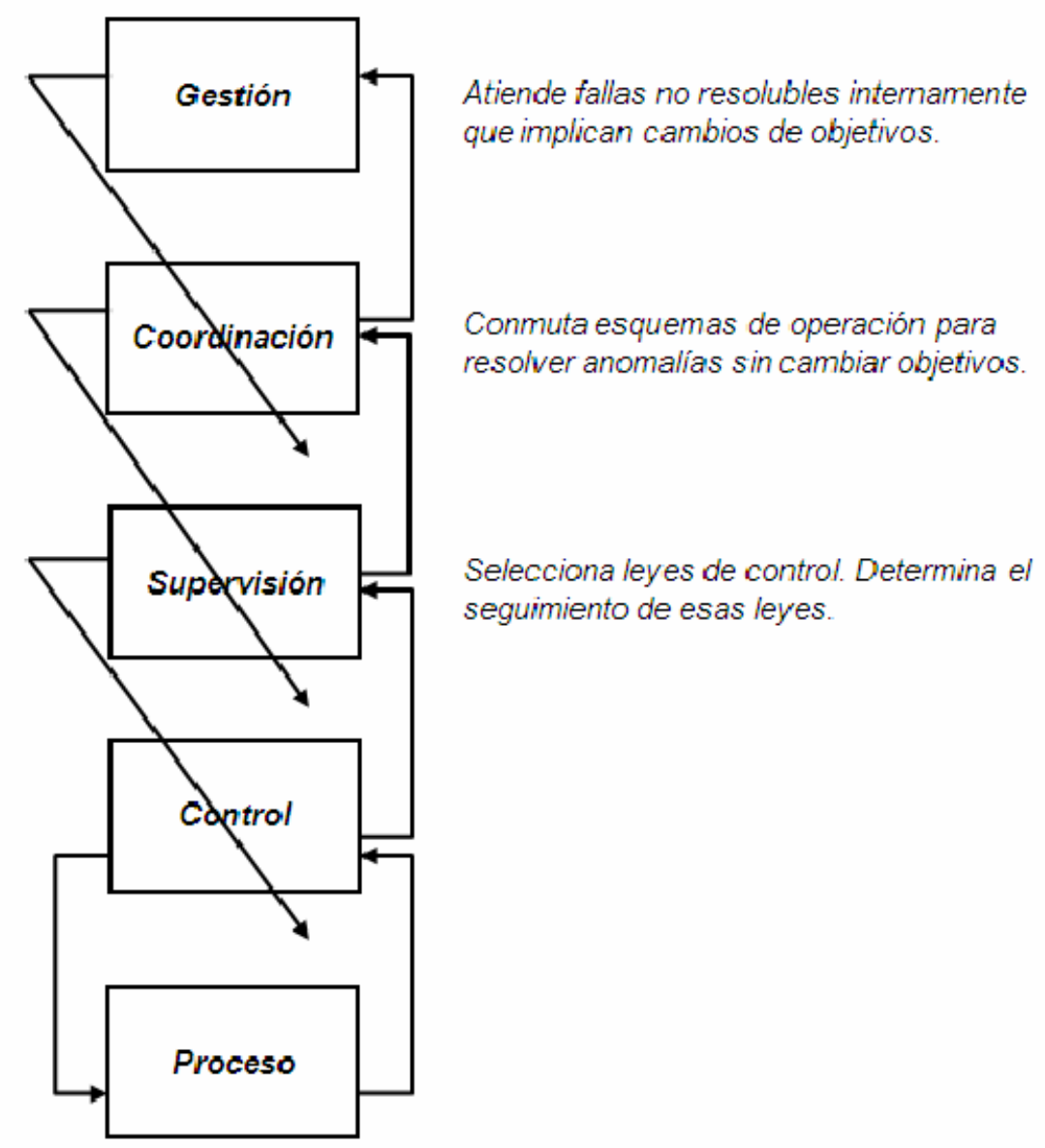

Fig. 3: Estructura jerárquica de la supervisión

\section{Definiciones Preliminares}

Para la representación de las dinámicas discretas se utilizan las redes de Petri generadoras de lenguajes (Peterson, 1981) o redes de Petri etiquetadas, apropiadas para realizar la síntesis del supervisor desde la propuesta de Ramage y Wonham (Ramadge y Wonham, 1984). Las dinámicas continuas son modeladas mediante redes de Petri diferenciales (Champagnat, et al., 1998; Villani, et al., 2004; Eigi Miyagi, et al., 2001). Desde el punto de vista matemático, el tratamiento de modelos discretos y continuos es complejo y dado que los modelos representan dos vistas diferentes de un sistema, podría ser más eficiente trabajar con dos modelos interactuantes separados. 


\section{Redes de Petri de Alto Nivel con Ecuaciones Diferenciales, DHLPN}

Formalmente una DHLPN es una tupla, $H=\left\langle P, T, A, M_{0}, \Sigma, l, \vartheta, C\right.$, Guard, $X, Z$, Flow, Init $\rangle$ Donde, $P=P_{D} \cup P_{C}$, donde $P_{C}$ son los estados que tienen ecuaciones diferenciales representando procesos continuos y $P_{D}$ son los estados discretos del sistema; $T$, es el conjunto de transiciones del proceso, además se define ' $t$ como el conjunto de eventos anteriores a la transición $t$; $A \subseteq(P \times T) \cup(T \times P)$ es un conjunto finito de arcos representando el flujo de relaciones; $v$ es un conjunto finito no vacío de tipos de datos $\left(\mathbb{R}, \mathbb{Z}_{;} \mathbb{R}^{+}, \mathbb{B}\right.$, String $) ; C$ es una función que mapea a cada lugar $P$ un tipo de datos, tal que $C: P \rightarrow \vartheta$, cabe aclarar que todos los estados que tienen ecuaciones diferenciales tienen como tipo de dato $\mathrm{R}$ y por eso no es necesario especificarlo; Guard relaciona una función de guarda que mapea a cada transición un predicado que representa un valor booleano de la forma, Guard:T $\rightarrow 2^{X \cup \vartheta} ; M_{0}$ es la función de inicialización; $\Sigma$ es un conjunto finito no vacío de símbolos de un alfabeto asociados con transiciones por el etiquetamiento, está dividido en dos conjunto disjuntos: conjunto de eventos controlables y no controlables, $\Sigma=\Sigma_{c} \cup \Sigma_{n c}$ (Cassandras y Lafortune, 2008); $l: T \rightarrow \Sigma \cup\{\varepsilon\}$ es una función de etiquetamiento; $X$, son las variables continuas, $X=\left\{x_{1}, x_{2}, \ldots, x_{n}\right\}, X \in \mathbb{R}^{n} ; Z$, es el conjunto de las variables discretas de entrada y de salida, $Z=\left\{z_{1}, z_{2}, \ldots, z_{n}\right\}, z_{n} \in\{0,1\}, Z=Z_{\text {in }} \cup Z_{\text {out }}$, donde $Z_{\text {in }}$ y $Z_{\text {out }}$ son las entradas y salidas binarias respectivamente; Flow, Matriz en la que se muestra la evolución de las variables, en el caso de las variables continuas se muestran las ecuaciones diferenciales (ED's) y en las discretas se muestra la evolución de la variable. Flow $=\left(\right.$ Flow $_{D} \cup$ Flow $\left._{C}\right)$, Flow $_{C}: P_{C} \times X \wedge\left(\right.$ Flow $\left._{C}: P_{C} \rightarrow F_{i}(X, \dot{X})=0\right)$

Flow $_{D}: P \rightarrow 2^{Z_{\text {out }}}, Z_{\text {outj }} \neq Z_{\text {out } j+1}$.

Es decir que la variable $z_{\text {out }}$ en el estado $j$ sea diferente a la misma variable en el estado $j+1$; Init $\subseteq P_{C} \times X$, son los valores iniciales de las variables continuas.

\section{Evolución y Dinámica de una DHLPN}

Para una DHLPN, se definirá el concepto de marca de la siguiente forma, $\forall H \exists M / M \supseteq M_{C} \cup M_{D}$. Donde, $\quad M_{D}: P_{D} \rightarrow \mathbb{N}, \quad M_{C}: P_{C} \rightarrow\{$ enable, disable $\} . \quad$ Si $M_{C}\left(q_{C i}\right)=\{$ enable $\}$ significa que las ecuaciones que estan en este lugar comienzan a evolucionar partiendo de las condiciones iniciales que se presentan en Init. Por efectos prácticos, se supone que los arcos de la red son de peso 0 ó 1.

Para que una transición se pueda disparar, es necesario que se cumpla la condición de sensibilización, para lo cual se presentan los siguientes casos:

Caso 1: La transición $t_{1}$ de la figura 4 se sensibiliza si, $\left[M_{C}\left(q_{C i}\right)=\{\right.$ enable $\left.\}\right] \wedge[$ guard $(t)=$ true $]$. Cuando $t_{1}$ se dispara la marca de $p_{c i}$ pasa a ser disable y la de $p_{c i+1}$ pasa a ser enable.

Caso 2: La transición $t_{1}$ de la figura 5 se sensibiliza si, $\left[M_{C}\left(q_{C i}\right)=\{\right.$ enable $\left.\}\right] \wedge\left[M_{D}\left(p_{D i}\right) \geq 1\right] \wedge[\operatorname{guard}(t)=$ true $],\left(p_{C i} \cup p_{D i}\right) \in^{\bullet} t$. Cuando la transición se dispara $p_{c i}$ se marca con disable, $p_{D i}$ pierde su marca y $p_{D i+1}$ queda marcada. 
Caso 3: La transición $t_{1}$ de la figura 6 se sensibiliza si, $\left[M_{D}\left(p_{D i}\right) \geq 1\right], p_{D i} \in^{*} t$, es decir en el caso en el que en la entrada a la transición se tienen dos estados discretos, la transición se habilita si en los dos estados hay marca. Cuando la transición se dispara, los dos estados anteriores a $t_{1}$ pierden su marca y el estado $p_{D i+1}$ pasa de disable a enable

\section{[guard]}

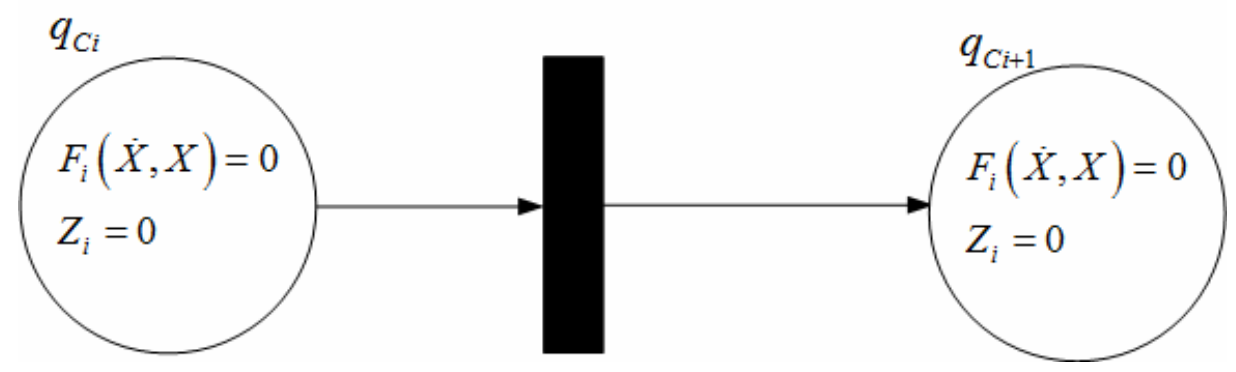

Fig. 4: DHLPN Caso 1

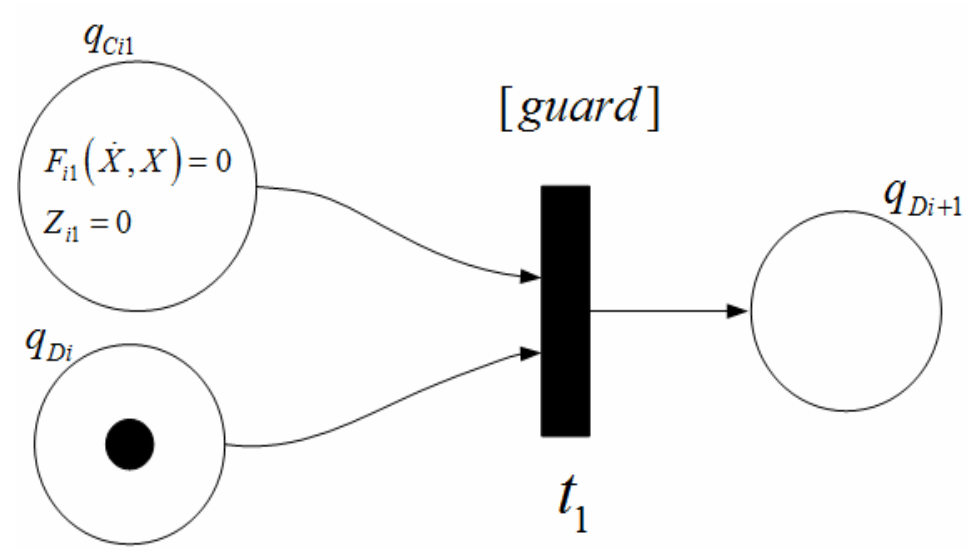

Fig. 5:DHLPN Caso 2

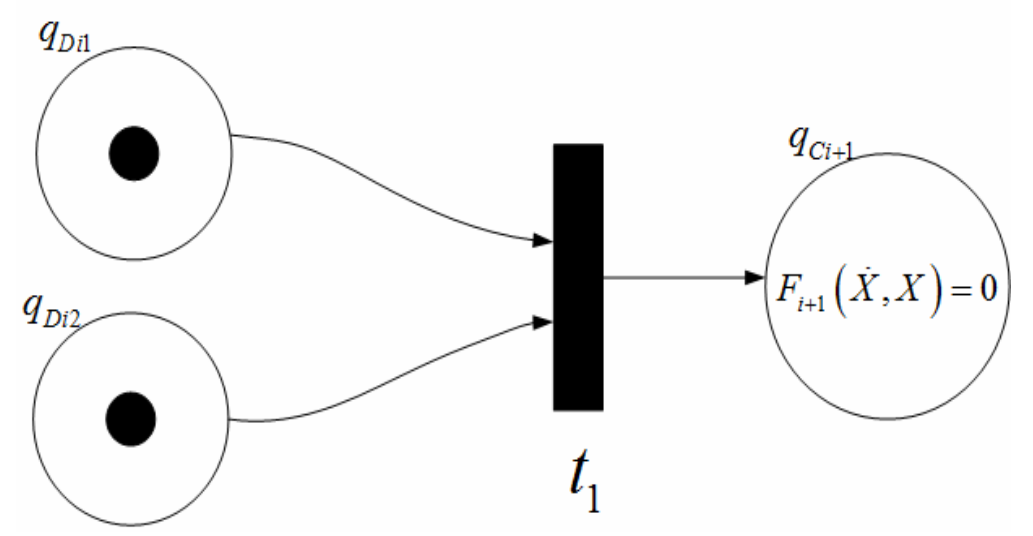

Fig. 6: DHLPN Caso 3

De esta manera se puede decir que la red de Petri sirve como supervisor de la parte continua del sistema, y al mismo tiempo las ecuaciones diferenciales (parte continua) controlan la evolución de la red de Petri (Champagnat, et al., 1998). 


\section{Arquitectura del sistema de supervisión}

La arquitectura propuesta para el sistema de supervisión incluye un bloque Detector de eventos (en la figura 7 se ve como $x / \sigma$ ) o cuantificador, que permite establecer de manera discretizada, la región del espacio de estados en la que opera el sistema; un Diagnosticador (en la figura 7 se ve como $\Gamma_{D}$ ), que establece las condiciones de falla y se obtiene desde señales de sensores de falla, a partir de técnicas de análisis de falla o desde módulos de diagnóstico.

Partiendo del cuantificador y del Diagnosticador se crea una imagen discreta del proceso, mediante un Autómata de la planta. Este autómata representa los estados relevantes del proceso y a partir de la observación de esos estados, el supervisor toma las decisiones que actúan sobre el controlador del proceso, conformando el sistema de supervisión que se presenta en la figura 8. La toma de decisiones puede ser efectuada por operadores humanos o por operadores con asistencia de sistemas inteligentes. El modelo del autómata de la planta se construye con base en los estados relevantes de la planta (modelado mediante autómatas híbridos o redes de Petri híbridas).

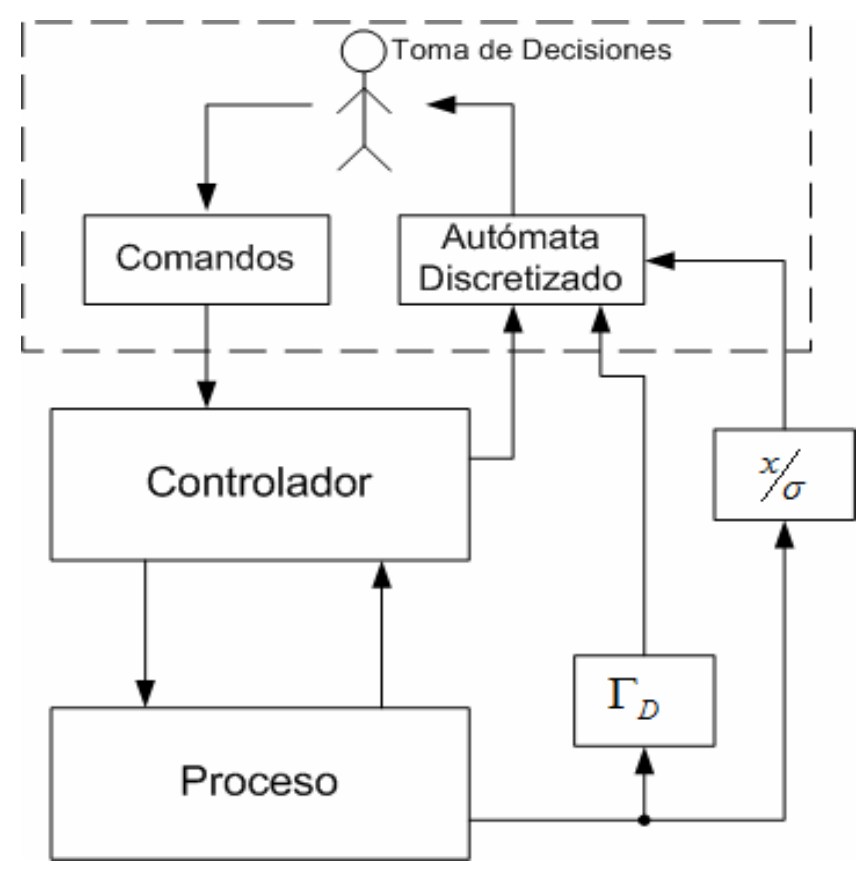

Fig. 7: Propuesta sistema de supervisión

\section{Especificación}

El punto de partida para iniciar el diseño del sistema de supervisión es la definición de las especificaciones para determinar las funcionalidades y el alcance del mismo. La expresión formal de las especificaciones, es la base para la construcción de los modelos del sistema de supervisión. Para su elaboración, deben tenerse en cuenta las intervenciones esperadas por parte del operador humano y las definidas para sistemas informáticos de apoyo a la toma de decisiones en el nivel de supervisión. Considerando que se ha seleccionado una base formal para expresar los modelos del comportamiento del proceso controlado y supervisado, las especificaciones se expresan en la misma base formal. La siguiente es una guía para la elaboración de las especificaciones. La representación formal de las mismas se ilustra en el ejemplo del numeral 6. 


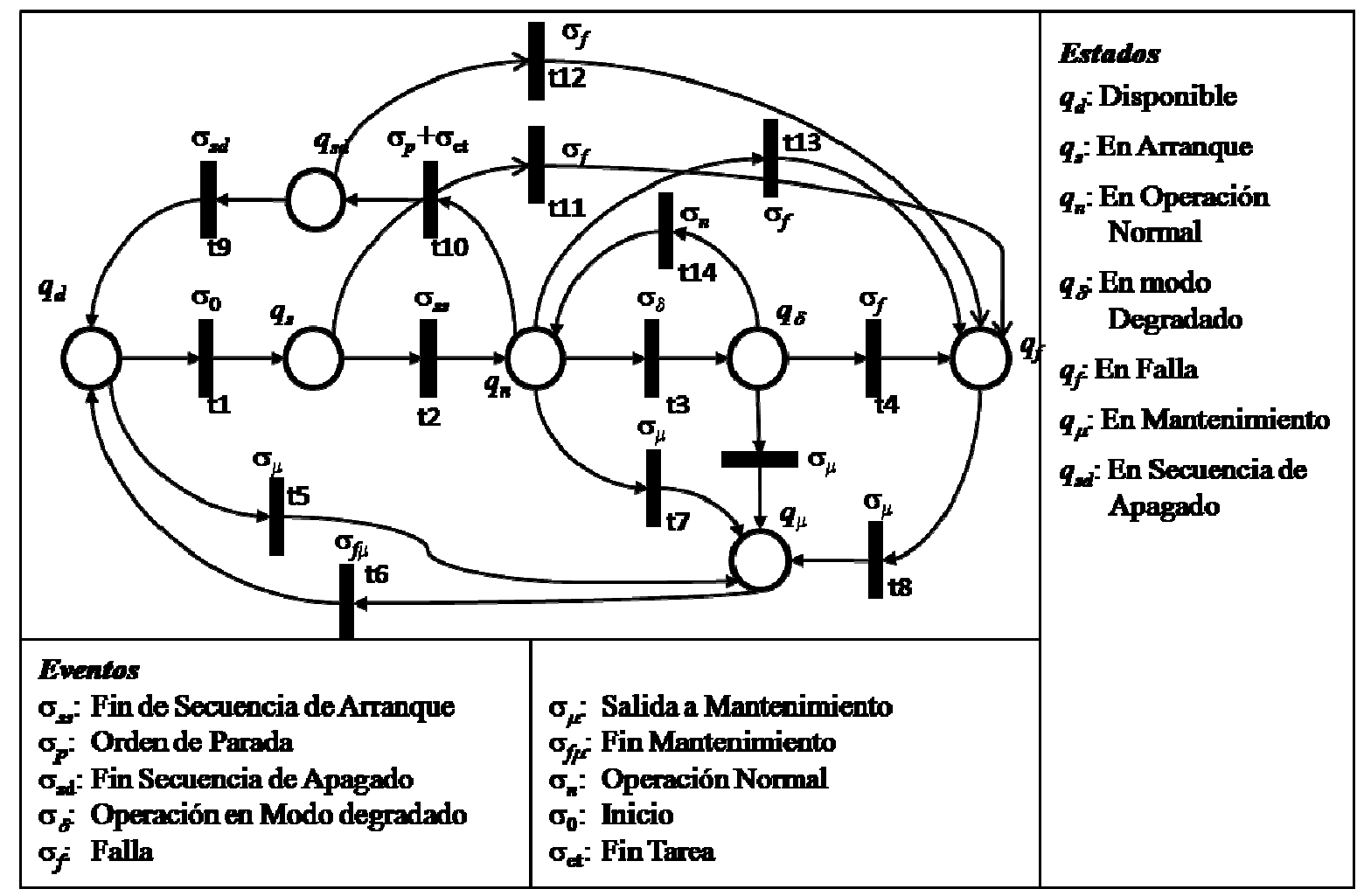

Fig. 8: Modelo del autómata

Paso 1. Determinar las acciones del operador. Se determinan mediante Redes de Petri de la forma $H=\left\langle P, T, A, M_{0}, \Sigma, l, \vartheta, C\right.$, Guard, $X, Z$, Flow $_{D}$, Init $\rangle$. Estas acciones se determinan tanto en operación normal, como en operación degradada y durante contingencias.

Paso 2. Determinar los estados relevantes del proceso. Se determina el conjunto $P_{\text {rel }}=\left\{p_{\text {rel } 1}, p_{\text {rel } 2}, \ldots, p_{\text {reln }}\right\}$. Además de estos estados, se determinan los macroestados, por ejemplo el macro estado "en proceso de arranque".

Paso 3. Determinar secuencias de arranque y parada. Se expresan mediante redes de Petri de la forma

$$
H_{0}=\left\langle P_{0}, T_{0}, A_{0}, M_{0}, \Sigma_{0}, l_{0}, \vartheta_{0}, C_{0}, \text { Guard }_{0}, X_{0}, Z_{0}, \text { Flow }_{D 0}, \text { Init }_{0}\right\rangle .
$$

Paso 4. Determinar las contingencias del sistema.

Paso 5. Definir las condiciones que determinan el estado de operación degradada.

Paso 6. Determinar las regiones de operación. $\Omega=\left\{\Omega_{1}, \Omega_{2}, \ldots, \Omega_{n}\right\}$

Paso 7. Determinar las leyes de control empleadas en cada región de operación y sus respectivos parámetros. Leyes de control: $U=\left\{U_{1}, U_{2}, \ldots, U_{n}\right\}$. Parámetro $u_{i j}$, parámetro $i$ de la ley de control $j$.

Paso 8. Determinar indicadores de desempeño. $\lambda=\left\{\lambda_{1}, \lambda_{2}, \ldots, \lambda_{n}\right\}$

Paso 9. Determinar límites operativos y restricciones operativas.

Enlace con la HMI 
La interfaz HMI (Human - Machine interface), sirve de intermediaria entre el operador humano y el autómata discretizado y entre el operador y el controlador. Una vez creado el autómata discretizado, se realiza un enlace entre sus estados y eventos y la interfaz, como se muestra en la figura 9.

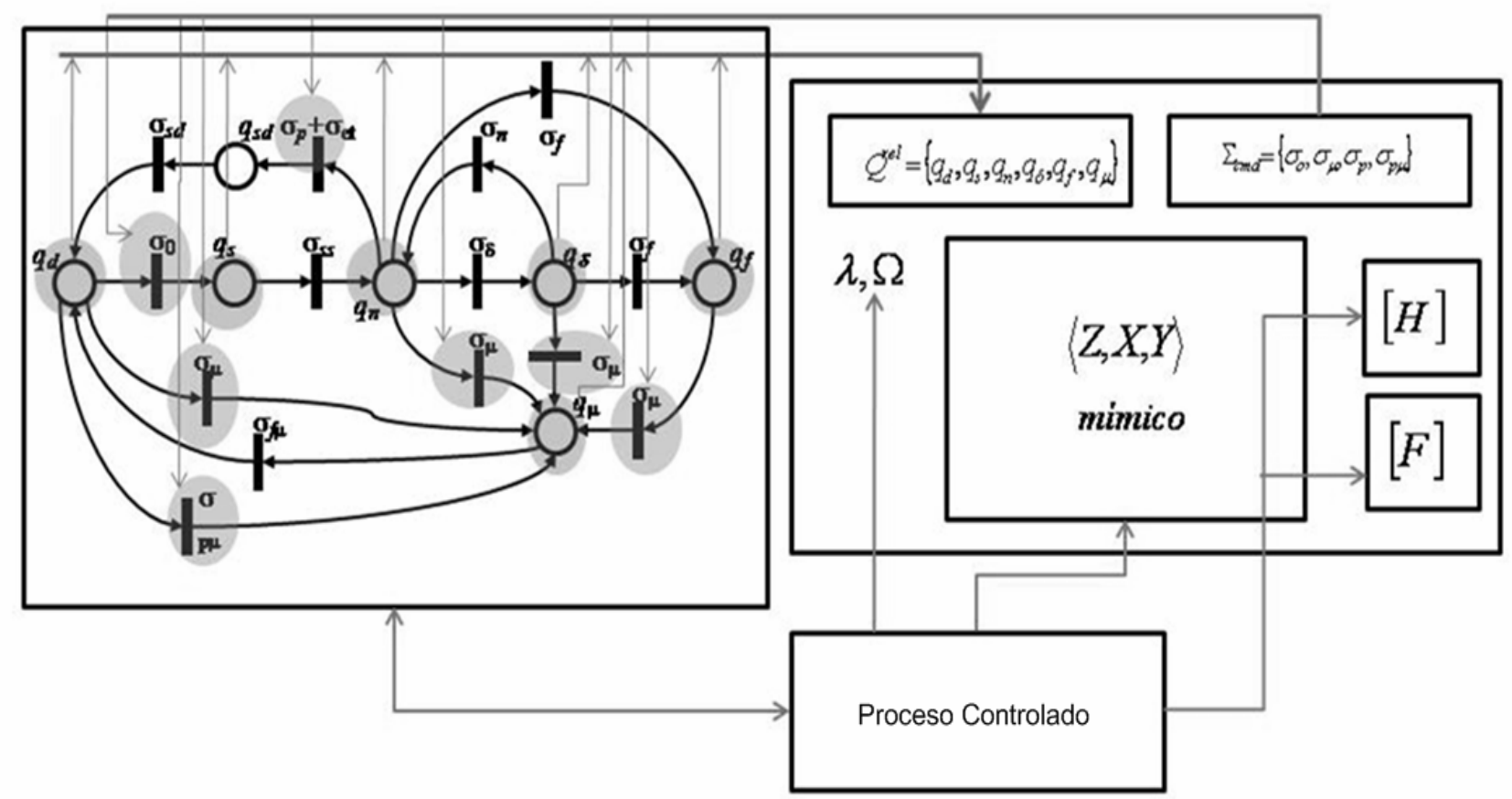

Fig. 9: Enlace con la HMI

\section{EJEMPLO DE APLICACIÓN}

Para ilustrar la metodología se planteará el arranque de la caldera que se ve en la figura 10, el procedimiento consiste en lo siguiente: primero se debe verificar que las válvulas de entrada de aire y de gas estén complemente cerradas además de la salida de vapor. Luego se debe abrir la entrada de agua hacia el tambor hasta que este esté lleno al 35\% de su capacidad máxima; cuando se llegue a este punto se abren las válvulas de aire y de gas hasta el valor mínimo en el cual se produce la combustión, en ese punto se enciende el transformador para generar la combustión y en el momento en que esta se genere se apaga el transformador y se abre la salida de vapor. El modelo formal mediante DHLPN de este proceso es el que se ven en la figura 11. Las especificaciones de todo el proceso, incluyendo el arranque se ven en las tablas 1 a la 12. Además en las figuras 12 y 13 se ve la HMI según el método propuesto en la sección 5.

Tabla 1: Variables de Estado

\begin{tabular}{|l|l|}
\hline$x_{1}$ & Presión demandada \\
\hline$x_{2}$ & Temperatura de la salida de gases \\
\hline$x_{3}$ & Posición válvula de combustible \\
\hline$x_{4}$ & Posición válvula de aire \\
\hline$x_{5}$ & Nivel de agua en el tanque \\
\hline$x_{6}$ & Presión del vapor entregado \\
\hline$x_{7}$ & Temperatura del vapor entregado \\
\hline
\end{tabular}




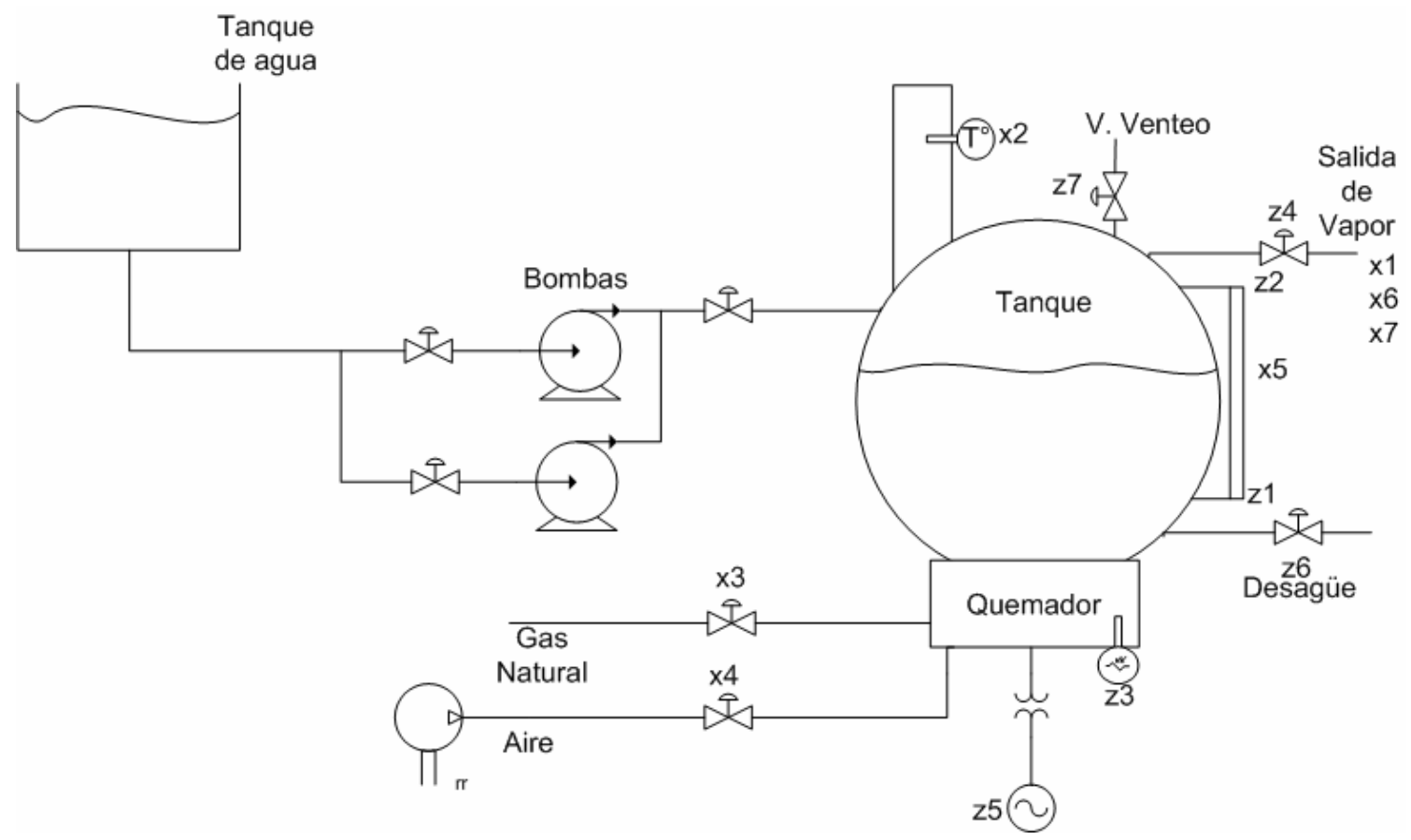

Fig. 10: Caldera

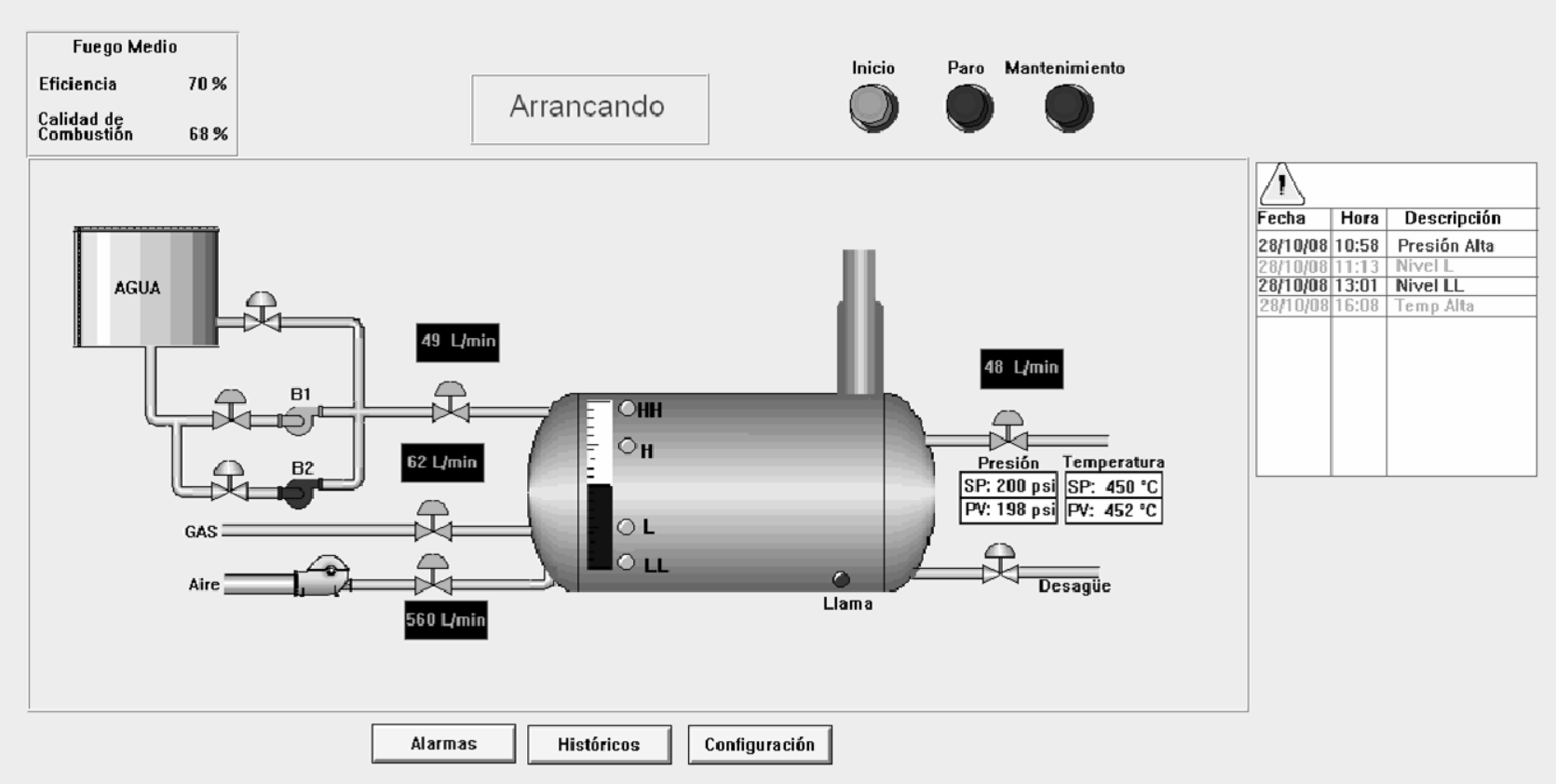

Fig. 11: Mapeo de la Interfaz gráfica 


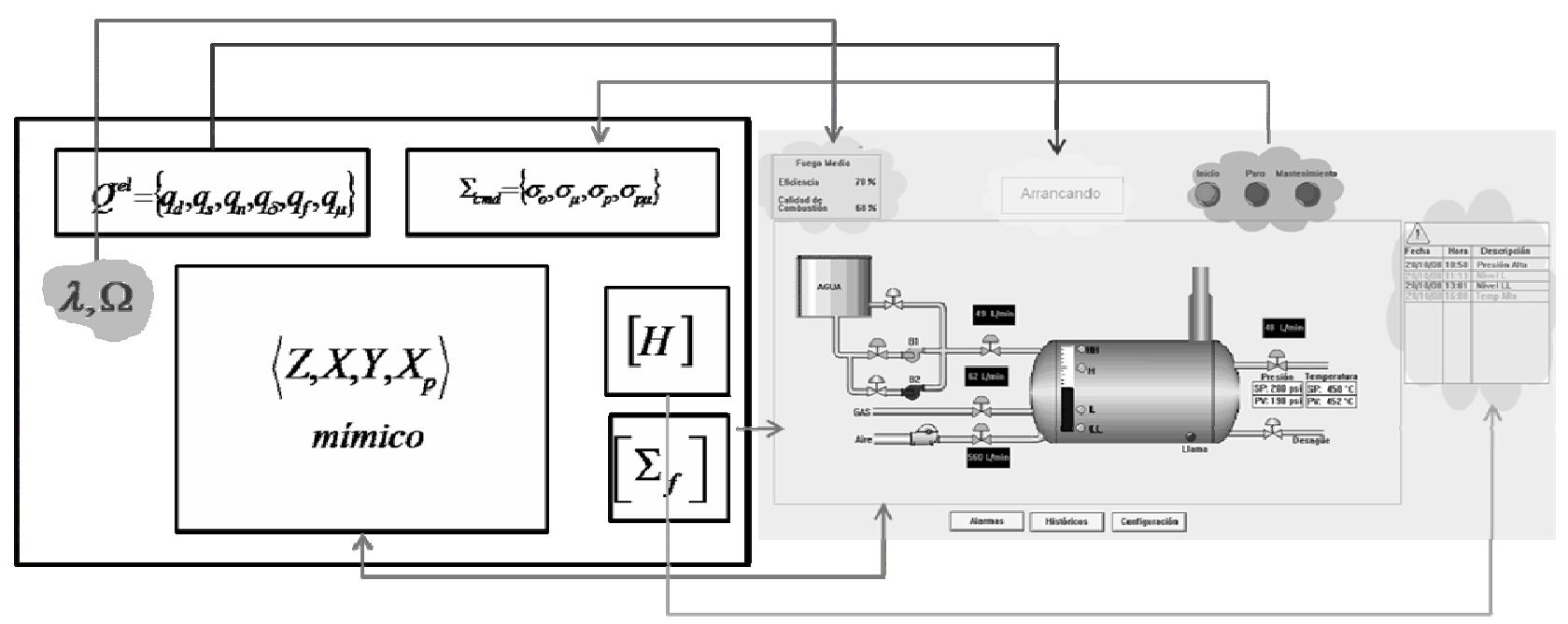

Fig. 12: HMI Caldera

Tabla 2: Variables Discretas

\begin{tabular}{|l|l|}
\hline$z_{1}$ & Nivel de agua bajo \\
\hline$z_{2}$ & Nivel de agua alto \\
\hline$z_{3}$ & Fotocelda \\
\hline$z_{4}$ & Válvula salida de vapor \\
\hline$z_{5}$ & Transformador \\
\hline$z_{6}$ & Válvula de desagüe del tanque \\
\hline$z_{7}$ & Válvula de venteo \\
\hline
\end{tabular}

Tabla 3: Variables de Entrada

\begin{tabular}{l|l}
\hline$u_{1}$ & Presión entregada
\end{tabular}

$u_{2}$ Posición válvula de combustible

\begin{tabular}{l|l}
$u_{3}$ & Posición válvula de aire \\
\hline
\end{tabular}

Tabla 4: Variables de Salida

\begin{tabular}{|l|l|}
\hline$y_{1}$ & Presión entregada \\
\hline$y_{2}$ & Posición válvula de combustible \\
\hline$y_{3}$ & Posición válvula de aire \\
\hline$y_{4}$ & Válvula de entrada de agua \\
\hline
\end{tabular}


Tabla 5: Parámetros del Proceso

\begin{tabular}{|c|l|}
\hline$x_{\max 3}$ & Posición máxima de la válvula de combustible \\
\hline$x_{\max 4}$ & Posición máxima de la válvula de aire \\
\hline$x_{\min 3}$ & Posición mínima para la válvula de combustible \\
\hline$x_{\min 4}$ & Posición mínima para la válvula de aire \\
\hline$x_{\max 5}$ & Valor máximo permitido para el nivel en el tanque \\
\hline$x_{\min 5}$ & Valor mínimo permitido para el nivel en el tanque \\
\hline$\tau_{e}$ & Tiempo de espera, varía según el estado del proceso \\
\hline$\tau_{c h}$ & Tiempo para que se de ignición \\
\hline $\begin{array}{l}\text { Nota: Los valores de } \\
\text { 0.95 y el1.05 del valor exacto del parámetro, para evitar los problemas propios de las variables } \\
\text { análogas, tales como la histéresis y las fluctuaciones propias de un sistema fluido. }\end{array}$ \\
\hline
\end{tabular}

Tabla 6: Alarmas

\begin{tabular}{|c|c|c|c|}
\hline \multirow[t]{2}{*}{ TAG } & \multirow[t]{2}{*}{ Descripción } & Prioridad & \multirow[t]{2}{*}{ Causa } \\
\hline & & $\mathrm{C} / \mathrm{A} / \mathrm{M} / \mathrm{B}$ & \\
\hline$h_{1}$ & Temperatura alta en los gases de combustión & $\mathrm{M}$ & $\sigma_{\delta 1}=\left\lfloor x_{2}>x_{p 2} \wedge t<\tau_{e}\right\rfloor$ \\
\hline$h_{2}$ & $\begin{array}{l}\text { Temperatura alta en los gases de combustión, } \\
\text { permanente }\end{array}$ & A & $\sigma_{f 1}=\left\lfloor x_{2}>x_{p 2} \wedge t>\tau_{e}\right]$ \\
\hline$h_{3}$ & Nivel en el tanque bajo & $\mathrm{M}$ & $\sigma_{\delta 2}=\left[x_{5}<L\right]$ \\
\hline$h_{4}$ & Nivel en el tanque bajo, crítico & $A$ & $\sigma_{f 2}=\left[x_{5}<L L\right]$ \\
\hline$h_{5}$ & Nivel en el tanque alto & $\mathrm{M}$ & $\sigma_{\delta 3}=\left[x_{5}>H\right]$ \\
\hline$h_{6}$ & Nivel en el tanque alto, crítico & A & $\sigma_{f 3}=\left[x_{5}>H H\right]$ \\
\hline$h_{7}$ & Falta de ignición & A & $\sigma_{f 4}=\left[t<\tau_{c h}\right]$ \\
\hline$h_{8}$ & Eficiencia baja & $\mathrm{M}$ & $\sigma_{\delta 4}=\left[0,8 k_{e}>y_{1} / y_{2}>1 \cdot 2 k_{e}\right.$ \\
\hline
\end{tabular}

Tabla 7: Fallas

\begin{tabular}{|l|l|}
\hline$h_{2}$ & $\begin{array}{l}\text { Temperatura alta en los gases de } \\
\text { combustión, permanente }\end{array}$ \\
\hline$h_{4}$ & Nivel en el tanque bajo, crítico \\
\hline$h_{6}$ & Nivel en el tanque alto, crítico \\
\hline$h_{7}$ & Falta de ignición \\
\hline$h_{1}$ & Subida transitoria de la temperatura en los gases de combustión \\
\hline$h_{3}$ & Nivel en tanque bajo, pero el sistema está trabajando en solucionar el problema \\
\hline
\end{tabular}


Tabla 8: Eventos que determinan operación degradada

\begin{tabular}{|c|l|}
\hline$h_{1}$ & $\begin{array}{l}\text { Subida transitoria de la temperatura en los } \\
\text { gases de combustión }\end{array}$ \\
\hline$h_{3}$ & $\begin{array}{l}\text { Nivel en tanque bajo, pero el sistema está } \\
\text { trabajando en solucionar el problema }\end{array}$ \\
\hline$h_{5}$ & $\begin{array}{l}\text { Nivel en tanque alto, pero el sistema está } \\
\text { trabajando en solucionar el problema }\end{array}$ \\
\hline$h_{8}$ & $\begin{array}{l}\text { La relación de presión del vapor y del } \\
\text { combustible se salió de la banda permitida }\end{array}$ \\
\hline
\end{tabular}

Tabla 9: Indicadores de desempeño

\begin{tabular}{|c|l|c|}
\hline Símbolo & Nombre & Descripción \\
\hline$\lambda_{1}$ & Eficiencia & $\lambda_{1}=y_{1} / y_{2}$ \\
\hline$\lambda_{2}$ & Mala combustión & $\lambda_{2}=x_{2} / x_{p 2}$ \\
\hline
\end{tabular}

Tabla 10: Regiones de Operación

\begin{tabular}{|c|l|l|}
\hline & Descripción & Región \\
\hline$\Omega_{1}$ & Fuego Bajo & $\left(0,5 x_{\max 1} \leq x_{1} \leq 0,7 x_{\max 1}\right) \wedge\left(0,5 x_{\max 3} \leq x_{1} \leq 0,7 x_{\max 3}\right)$ \\
\hline$\Omega_{2}$ & $\begin{array}{l}\text { Fuego } \\
\text { Medio }\end{array}$ & $\left(0,7 x_{\max 1} \leq x_{1} \leq 0,9 x_{\max 1}\right) \wedge\left(0,7 x_{\max 3} \leq x_{1} \leq 0,9 x_{\max 3}\right)$ \\
\hline$\Omega_{3}$ & Fuego Alto & $\left(0,9 x_{\max 1} \leq x_{1} \leq 1,0 x_{\max 1}\right) \wedge\left(0,9 x_{\max 3} \leq x_{1} \leq 1,0 x_{\max 3}\right)$ \\
\hline
\end{tabular}

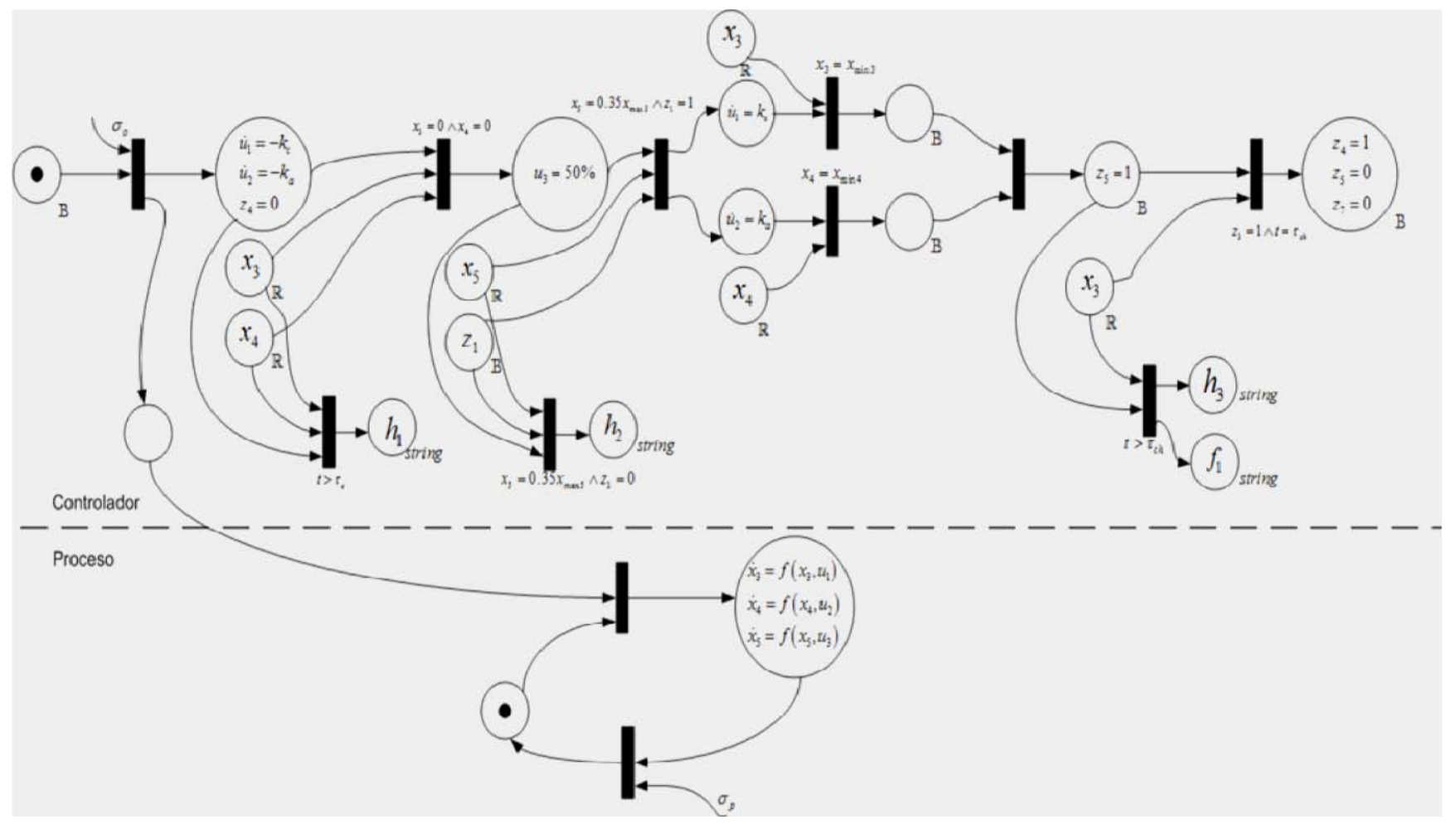

Fig. 13: Modelo Formal 


\section{CONCLUSIONES}

Como un paso previo para abordar el diseño de sistemas de supervisión desde el enfoque holónico, se presentan algunas bases metodológicas que serán útiles al momento de concebir la supervisión mediante una descentralización del conocimiento, los modelos del proceso y la toma de decisiones. Inicialmente se expresa el comportamiento del sistema desde una base formal unificada. Las redes de Petri han resultado ser apropiadas para este fin, ya que capturan las evoluciones discretas del proceso continuo; acoplan las dinámicas continuas con las discretas de los niveles de decisión y permiten la síntesis del supervisor desde las técnicas del control supervisorio, a partir del análisis del árbol de alcanzabilidad, obtenido de la ejecución de la PN como generadora de lenguajes. Para modelar el comportamiento deseado del sistema de supervisión, se hace una propuesta para el levantamiento de las especificaciones del mismo y expresarlo mediante la misma base formal unificada. Se hacen también algunos planteamientos para relacionar los aspectos teóricos con los aspectos tecnológicos de la implementación. La continuidad de esta trabajo se orienta al diseño de sistemas de supervisión desde el enfoque holónico, utilizando técnicas de síntesis de la teoría de control supervisorio.

\section{AGRADECIMIENTOS}

Los resultados de este trabajo hacen parte de los proyectos "Arquitectura Holónica de Bajo Costo para la Supervisión, Integración y Control de sistemas de Producción" financiado por DINAIN (Dirección Nacional de Investigaciones de la Universidad Nacional) y "Aplicabilidad del Modelo de Integración Holónico en sistemas a eventos discretos granulares con observabilidad limitada" financiado por Colciencias. Ambos proyectos cuentan además con el apoyo de la Universidad de los Andes, Venezuela.

\section{REFERENCIAS}

Akesson, K. y M. Fabian, Implementing supervisory control for chemical batch processes, Proceedings of the 1999 IEEE International Conference on Control Applications, Vol. 2, 1272-1277, (1999).

Alvarez, A., W. García, O. Camacho y E. Chacón, Automation of a steam generation plant using hybrid systems, ISA Transactions, 38(1), 87-99, (1999).

Avouris, N.M., M.H. Van Liedekerke, G.P. Lekkas y L.E. Hall, User interface design for cooperating agents in industrial process supervision and control applications, International Journal of Man-Machine Studies, 38(5), 873-890, (1993).

Basile, F. y P. Chiacchio, On the Implementation of Supervised Control of Discrete Event Systems, IEEE Transactions on Control Systems Technology, 15(4), 725-739, (2007).

Blanke, M., M. Staroswiecki y N.E. Wu, Concepts and methods in fault-tolerant control, Proceedings of the 2001American Control Conference, Vol. 4, 2606-2620, (2001).

Boyer, S.A., SCADA: supervisory control and data acquisition, ISA, The Instrumentation Systems and Automation Society, (2004).

Cassandras, C. y S. Lafortune, Introduction to discrete event systems, Springer Science+Business Media, (2008).

Champagnat, R., P. Esteban, H. Pingaud y R. Valette, Petri net based modeling of hybrid systems, Computers in Industry, 36(1-2), 139-146, (1998).

Charbonnier, F., H. Alla y R. David, Discrete-event dynamic systems, IEEE Transactions on Control Systems Technology, 7(2), 175-187, (1999).

Contreras, O., J.L. De La Rosa y J.M. Frigola, I-SS: integrated supervision systems approach based on interactive components, Proceedings of the 2001 8th IEEE International Conference on Emerging Technologies and Factory Automation, Vol. 2, 119-127, (2001).

Da Silveira, M.R. y M. Combacau, Supervision and control of heterarchical discrete event systems, 
Sba: Controle \& Automação Sociedade Brasileira de Automatica, 171-9, (2006).

Eigi Miyagi, P., E. Villani y N. Maruyama, Modeling of hybrid supervisory systems using UML and Petri nets, 2001 8th IEEE International Conference on Emerging Technologies and Factory Automation, Vol. 2, 149-158, (2001).

Esteva Payet, S., Modelling, control and supervision for a class of hybrid system, Tesis de Maestría, Universitat de Girona, Barcelona, España, (2003).

Fiol-Roig, G. y M. Ferrer-Gili, Expert system for supervision of real time control processes, Computational Cybernetics and Simulation, Vol. 2, 1966-1971, (1997).

Garcia, H.E., A hierarchical platform for implementing hybrid systems in process control, Control Engineering Practice, 5(6), 779-789, (1997).

Gonnet, S. y O. Chiotti, Modeling of the supervisory control system of a multipurpose batch plant, Computers \& Chemical Engineering, 23(4-5), 611-622, (1999).

IEEE 100, The Authoritative Dictionary of IEEE Standards Terms, $7^{\circ}$ Ed., (2000).

Isermann, R., Supervision, fault-detection and fault-diagnosis methods - An introduction, Control Engineering Practice, 5(5), 639-652, (1997).

Isermann, R., On fuzzy logic applications for automatic control, supervision, and fault diagnosis, IEEE Transactions on Systems, Man and Cybernetics, Part A: Systems and Humans, 28(2), 221-235, (1998).

Izadi-Zamanabadi, R. y M. Blanke, A ship propulsion system as a benchmark for fault-tolerant control, Control Engineering Practice, 7(2), 227-239, (1999).

Jantzen, J., Fuzzy Supervisory Control, Informe técnico, Technical University of Denmark, (1998).

Kowalewski, Engell, Preußig y Stursberg, Verification of Logic Controllers for Continuous Plants Using Timed Condition/Event-System Models, Automatica, Vol. 35, 505-518, (1999).

Kwang-Hyun, C. y L. Jong-Tae, Synthesis of fault-tolerant supervisor for automated manufacturing systems: a case study on photolithographic process, IEEE Transactions on Robotics and Automation, 14(2), 348-351, (1998).

Lambert, M., B. Riera y G. Martel, Application of functional analysis techniques to supervisory systems, Reliability Engineering and System Safety, Vol. 64, 209-224, (1999).

Lo, C.H., Y.K. Wong y A.B. Rad, Intelligent system for process supervision and fault diagnosis in dynamic physical systems, IEEE Transactions on Industrial Electronics, 53(2), 581-592, (2006).

Lunze, J., B. Nixdorf y H. Richter, Process supervision by means of a hybrid model, Journal of Process Control, 11(1), 89-104, (2001).

Montmain, J. y S. Gentil, Causal modeling for supervision, Proceedings of the 1999 IEEE International Symposium on Intelligent Control/Intelligent Systems and Semiotics, 231-236, (1999).

Morgenstern, A. y K. Schneider, Synthesizing Deterministic Controllers in Supervisory Control, Informatics in Control, Automation and Robotics II, Springer Netherlands, 95-102, (2007).

Oltean, V., T. Borangiu y M. Manu, The Supervision of Hybrid Control Systems - A Layered Architecture, Computer Aided systems Theory, Vol. 1798/2000, 573-587, (2000).

Peterson, J.L., Petri Net Theory and the Modeling of Systems, Prentice Hall PTR, (1981).

Puig, V. y otros 4 autores, Control Tolerante a Fallos (Parte II): Mecanismos de Tolerancia y Sistema Supervisor, Revista iberoamericana de automática e informática industrial (RIAI), 1(2), 5-21, (2004).

Quek, C. y P.W. Ng, Fuzzy integrated process supervision, Proceedings of the 1996 IEEE International Symposium on Intelligent Control, 319-324, (1996). 
Quek, C. y A. Wahab, Real-time integrated process supervision, Engineering Applications of Artificial Intelligence, 13(6), 645-658, (2000).

Raisch, J. y S.D. O'Young, Discrete approximation and supervisory control of continuous systems, IEEE Transactions on Automatic Control, 43(4), 569-573, (1998).

Ramadge, P. y W. Wonham, Supervisory control of a class of discrete event processes, Analysis and Optimization of Systems, Vol. 63, 475-498, (1984).

Rezg, N., G. Neubert y J. Favrel, Proactive supervision control in discrete event systems using Petri nets, 1997 6th International Conference on Emerging Technologies and Factory Automation Proceedings, 275-280, (1997).

Rezg, N. y E. Niel, Extension of the supervision concept to the monitoring of discrete event systems, 1996 IEEE Conference on Emerging Technologies and Factory Automation Proceedings, Vol. 1, 150156, (1996).

Saridis, G. y K. Valavanis, Mathematical formulation of the organization level of an intelligent machine, 1986 IEEE International Conference on Robotics and Automation. Proceedings, Vol. 3, 267-272, (1986).

Saridis, G.N., Entropy formulation of optimal and adaptive control, IEEE Transactions on Automatic Control, 33(8), 713-721, (1988).

Simão, J.M., A contribution to the development of a HMS simulation tool and proposition of a metamodel for holonic control, Tesis de Doctorado, Henry Poincaré University, Curitiba, Brazil, (2005).

Stobart, R.K. y N.R. Shadbolt, Process Control Supervision Using Qualitative Models, Proceedings of 3rd International Conference on industrial and Engineering Applcications of Al and Expert Systems, ACM Press, (1990).

Suárez, S., B. López y J. Melendez, Towards holonic multiagent systems: Ontology for supervision tool boxes, Workshop de Agentes Inteligentes en el tercer milenio, 1-8, (2003).

Uraikul, V., C.W. Chan y P. Tontiwachwuthikul, Artificial intelligence for monitoring and supervisory control of process systems, Engineering Applications of Artificial Intelligence, 20(2), 115-131, (2007).

Vagelatos, G.A., G.G. Rigatos y S.G. Tzafestas, Incremental fuzzy supervisory controller design for optimizing the injection molding process, Expert Systems with Applications, 20(2), 207-216, (2001).

Villa, J.L., M. Duque, A. Gauthier y N. Rakoto-Ravalontsalama, Supervision and optimal control of a class of industrial processes, Vol. 2, 177-180 vol.2, (2003).

Villani, E., J.C. Pascal, P.E. Miyagi y R. Valette, Object oriented approach for cane sugar production: modelling and analysis, Control Engineering Practice, 12(10), 1279-1289, (2004).

Waissman, J. y otros 4 autores, Wastewater treatment process supervision by means of a fuzzy automaton model, Proceedings of the 2000 IEEE International Symposium on Intelligent Control, 163168, (2000).

Wang, W., H. Li y J. Zhang, A hybrid approach for supervisory control of furnace temperature, Control Engineering Practice, 11(11), 1325-1334, (2003).

Ya-Li, W., L. Ding y Z. Jian-Chao, Hybrid Petri nets of scheduling and supervisory control integrated system in process industry, Vol. 2, 1008-1013 vol.2, (2004).

Yazdi, H., Control and Supervision of Event-Driven Systems, Tesis de Doctorado, Technical University of Denmark (DTU), Denmark, (1997). 